\title{
Migraine exacerbation during Ramadan fasting
}

\author{
Ibrahim Abu-Salameh • Ygal Plakht • \\ Gal Ifergane
}

Received: 20 May 2010/Accepted: 5 July 2010/Published online: 22 July 2010

(C) Springer-Verlag 2010

\begin{abstract}
Fasting for approximately 1 month is an obligatory practice for Muslims during the month of Ramadan. We attempted to evaluate the effect of the Ramadan fasting on the frequency of migraine attacks among observant Muslim migraine sufferers using a cohort cross-over study. Thirty-two observant Muslim migraine sufferers filled a migraine diary during the Ramadan month and the following month (control). Two patients failed to complete the fast because of migraine suffering. During the Ramadan month, the patient expressed $9.4 \pm 4.3$ migraine days in average (range 3-20) when compared with $3.7 \pm 2.1$ migraine days in average (range 1-10) during the control month $(p<0.001)$. This phenomenon was associated with longer duration of migraine, lower frequency of migraine attacks, and migraine with aura. It seemed to be less pronounced in patients experiencing throbbing headaches and in married patients. Previous prophylactic therapy did not dampen this. Physicians treating Muslim migraine patients should discuss potential Ramadan month exacerbation with their patients and provide counseling regarding the prevention of dehydration and caffeine withdrawal. Pharmacological prophylactic treatment should be considered.
\end{abstract}

I. Abu-Salameh · G. Ifergane $(\bowtie)$

Headache Clinic, Department of Neurology, Soroka University

Medical Center, Beer-Sheva, Israel

e-mail: galif@clalit.org.il

\section{Y. Plakht}

Unit of Nursing Research, Soroka University Medical Center, Beer-Sheva, Israel

\section{Y. Plakht}

The Leon and Matilda Recanati School, Ben-Gurion University of the Negev, Beer-Sheva, Israel
Keywords Migraine $\cdot$ Ramadan $\cdot$ Fasting $\cdot$ Muslims · Beduins

\section{Introduction}

Fasting, the act of willingly abstaining from some or all food, drink, or both, is practiced to some extent by most religions. In Islam, fasting for approximately 1 month is an obligatory practice during the holy month of Ramadan [1], from fajr (dawn), until the maghrib (dusk). Muslims are prohibited from eating, drinking (including water), engaging in sexual activity, becoming angry, and smoking while fasting. Fasting in the month of Ramadan is one of the Pillars of Islam, and thus one of the most important acts of Islamic worship.

Migraine prevalence is estimated [2] to be approximately $12 \%$ of adults. Islam is the world's second largest religion after Christianity. According to a 2009 demographic study, Islam has 1.57 billion adherents [3], making up $23 \%$ of the world population. Therefore, approximately 90 million Muslims suffer from migraine.

Fasting was previously reported [4] to induce headache. Based on our clinical experience and observation, we attempted to evaluate the effect of the Ramadan fasting on the frequency of migraine attacks among observant Muslim migraine sufferers.

\section{Methods}

Set-up

A neurology clinic practicing in Bedouin settlements in the Negev desert area, of south Israel, operated by the only Bedouin Neurologist in Israel. 


\section{Patients}

Patients suffering from episodic migraine with and without aura (according to the International Classification of Headache Disorders 2nd edition (ICHD-II) [5]) treated in the neurological clinic.

Inclusion criteria: age $>18$ years, Muslim religion, observing the Ramadan fast, suffering from migraine with or without aura for more than 1 year (without any other headache type), with an average frequency of migraine attacks of 2-14 per month during 3 months prior to the study period, who completed a clinical workup to exclude secondary headache etiology.

Exclusion criteria: intracranial disorder, other than migraine, head trauma in the previous year, pregnancy or lactation during study period, inability (according to the investigators judgment) to fill a headache diary.

Patients who did not complete the diary, or did not complete the entire month of fasting were excluded from the study.

Protocol

\section{Inclusion visit}

Was preformed 7-14 days prior to the Ramadan month. The patients were asked to fill a questionnaire regarding personal data and headache history, allowing a diagnosis of migraine with or without aura according to the ICHD-II. During the visit, headache diaries were handed to the patients and they were instructed how to fill them.

\section{Ramadan headache diary}

A diary was filled by the patients during the Ramadan month. In the diary, the patients were instructed to record headache attacks similar to those they usually suffer. Patients were instructed to contact their physician regarding other types of headaches. The diary was returned to the investigator up to 10 days following the end of the month.

\section{Control headache diary}

A second diary, identical to the first, was given to the patients in the same visit. The diary covered a 30-day period beginning immediately following. The diary was returned to the investigator up to 10 days following the end of the control month.

The study protocol was approved by the Soroka University Medical Center Institutional Review Board.

\section{Statistical analysis}

All study parameters were presented as dichotomous or numeric data. The percent of categories (for dichotomous variables) and mean \pm standard deviation and range (for numeric variables) were presented. The comparison in number of migraine days between the Ramadan and the control periods was performed using Spearman's correlation and Wilcoxon's non-parametric test. For each patient, the ratio of migraine days during the Ramadan and control periods (called "Ramadan/Control Ratio") was calculated; yet, we assumed the normal distribution of the Ramadan/ Control Ratio variable, the comparison of rates in accordance to the study parameters was performed using Pearson correlation (numeric variables) and Mann-Whitney $U$ nonparametric test (dichotomous variables). The multivariable analysis used for determination of the independent predictors for rate increase was linear regression model. For each test, $p$ values (two-tailed) $<0.05$ were considered statistically significant.

\section{Results}

Thirty-two patients were enrolled to the study. Two patients discontinued the fast during the first week of Ramadan due to severe migraine attacks, and were excluded. Thirty patients completed the entire study protocol. No patients contacted to report unfamiliar headache symptoms.

The mean age of the patients was $34.4 \pm 10$, range 18-52. About three quarters of the patients were women $(73.3 \%)$. Half of the patients were employed, and $86.6 \%$ were married. About quarter of patients $(23 \%)$ resided in traditional Bedouin villages and camps, while the rest resided in semi-urban settlements.

The migraine characteristics of the patients included in the study are presented in Table 1.

During the Ramadan month, the patient expressed $9.4 \pm 4.3$ migraine days in average (range 3-20), compared with $3.7 \pm 2.1$ migraine days in average (range $1-10)$ during the control month $(p<0.001)$ (see Fig. 1). Positive correlation between the number of migraine days during the study periods was found $\left(R_{\mathrm{s}}=0.465\right.$; $p=0.01$ ).

On average, the number of migraine days during the Ramadan month was about three times gather than the control period (average of Ramadan/Control Ratio $=2.9 \pm 1.2$, range $0.6-5$ ).

The results of comparison of the Ramadan/Control Ratios between the study variables are presented in Table 2.

The increase in Ramadan/Control Ratio was not affected by age or gender. Table 3 presents the results of multivariate analysis determining factors associated with higher Ramadan/Control Ratio. The adjusted $R^{2}$ of the model was 0.567 . 
Table 1 Migraine features of the study population $(n=30)$

\begin{tabular}{ll}
\hline Migraine duration (years) & $15 \pm 8.85 ; 3-35$ \\
Migraine attack duration (h) & $27 \pm 12.5 ; 10-48$ \\
Pain severity & $8 \pm 0.97 ; 7-10$ \\
Unilateral & 60 \\
Throbbing & 56.6 \\
Increased by activity & 100 \\
Interfere with regular activity & 100 \\
Nausea or vomiting & 73.3 \\
Sensitivity to light & 96.7 \\
Sensitivity to sound & 96.7 \\
Aura & 26.7 \\
Acute treatment by triptans & 73.3 \\
Prophylactic treatment & 33.3 \\
\hline
\end{tabular}

The data presented as mean \pm standard deviation; range or percents

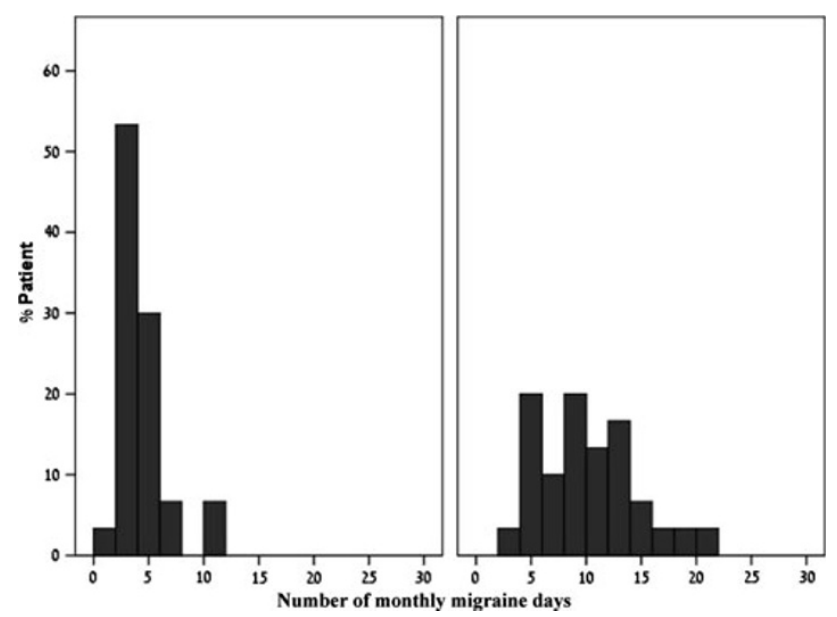

Fig. 1 Distribution of monthly migraine days during the Ramadan and the control months

\section{Discussion}

The occurrence of headache during fasting [4] is well known in the public and was established in the medical literature, especially with regard to short-term fasting such as the Yom-Kippur [6, 7] fast and the first day of Ramadan [8]. It seems to be associated with dehydration and caffeine withdrawal, and to be more common among sufferers of primary headaches. It is possible that other factors associated with changes in life style and stress also contribute to this phenomenon.

In the present cohort cross-over study; in which, we tried to evaluate the impact of Ramadan fast on the frequency of migraine attacks. We observed that a threefold increase in the frequency of migraine attacks during the entire month of Ramadan fasting, in a cohort of 30 episodic migraine patients treated in a neurological clinic. This phenomenon was associated with longer duration of migraine, lower frequency of migraine attacks, and migraine with aura. It seemed to be less pronounced in patients experiencing throbbing headaches and in married patients. Previous prophylactic therapy did not dampen this phenomenon.

Our study was preformed in 2009, in which Ramadan occurred in August, in the Negev desert area and dehydration is highly prevalent. Coffee and strong tea consumption is highly abundant in Bedouin culture and withdrawal is probably prominent. A previous [9] study has demonstrated higher rates of headache-related emergency room visits during Ramadan in Muslim communities in India. In addition, no epidemiological migraine surveys were preformed in the Bedouin community, our clinical experience is that migraine prevalence and characteristics are similar among Bedouins to the Jewish community. Therefore, we assume that increased frequency of migraine attacks among sufferers during the Ramadan is universal and also maybe to a lesser degree. Similar studies in cold weather and different lifestyles would provide us with further perspectives.

The International Classification of Headache, 2nd edition (ICHD-II, 2004) codes "Fasting headache" as a separated entity (10.5 of ICHD-II) [5]. Nevertheless, the ICHD-II criteria define fasting headache only when the patient was fasting for more than $16 \mathrm{~h}$. The fasting time, during the time period between sunset and sundown in Israel during the study month is $<14 \mathrm{~h}$, making it impossible to consider the headache attacks "fasting headache". However, it is possible that some of the headache attacks were similar to fasting headache in their pathophysiological mechanisms.

Our results raise an important clinical issue, involving the management of observant Muslim patients suffering from episodic migraine. Although none of our patients developed chronic migraine following the Ramadan fast, migraine suffering was dramatically increased and affected the patients' quality of life and their ability to practice their religious fate.

Physicians treating Muslim migraine patients should discuss the issue with their patients and ask about the patients previous Ramadan experiences, and adapt the patient management accordingly.

Till today no trial results were reported, it seems reasonable to advice the patients (especially in hot weather) to consume high quantities of water each night, and to minimize the consumption of caffeine during Ramadan nights to avoid dehydration and withdrawal.

In high risk patients (especially those with a history of Ramadan-associated migraine exacerbations), pharmacological treatment should be discussed with the patients. The treatment should preferably be once daily (because of the Ramadan schedule) and without long titration periods. 
Table 2 Univariate analysis: relationship of Ramadan/ Control Ratio with the study parameters
${ }^{a}$ Ramadan/Control Ratio calculated as ratio of migraine days during the Ramadan and control periods

Table 3 Multivariate analysis: factors associated with higher Ramadan/Control Ratio

\begin{tabular}{|c|c|c|c|c|c|}
\hline \multirow[t]{2}{*}{ Variable } & \multicolumn{3}{|c|}{ Ramadan/Control Ratio ${ }^{\mathrm{a}}$ (mean $\pm \mathrm{SD}$ ) } & & \multirow[t]{2}{*}{$P$ value } \\
\hline & Yes & & No & & \\
\hline Gender, male & $3.4 \pm 1.0$ & & $2.7 \pm 1.2$ & & 0.171 \\
\hline Throbbing headache & $2.6 \pm 1.1$ & & $3.3 \pm 1.2$ & & 0.094 \\
\hline Aura & $2.9 \pm 1.2$ & & $2.9 \pm 1.2$ & & 0.944 \\
\hline Unilateral headache & $2.9 \pm 1.0$ & & $2.9 \pm 1.5$ & & 0.983 \\
\hline Prophylactic therapy & $2.5 \pm 1.1$ & & $3.1 \pm 1.2$ & & 0.259 \\
\hline Triptan use & $2.9 \pm 1.0$ & & $3.0 \pm 1.7$ & & 0.906 \\
\hline Traditional Bedouin village & $2.9 \pm 1.5$ & & $2.9 \pm 1.1$ & & 0.748 \\
\hline Employment & $3.0 \pm 1.0$ & & $2.8 \pm 1.3$ & & 0.587 \\
\hline Married & $2.9 \pm 1.2$ & & $3 \pm 1.0$ & & 0.951 \\
\hline Variable & \multicolumn{3}{|c|}{ Correlation value $(r)$} & $P$ value & \\
\hline Age & \multicolumn{3}{|c|}{0.173} & 0.362 & \\
\hline Migraine years & \multicolumn{3}{|c|}{0.192} & 0.309 & \\
\hline Migraine attack duration & \multicolumn{3}{|c|}{-0.027} & 0.886 & \\
\hline Headache intensity (VAS) & \multicolumn{3}{|c|}{-0.275} & 0.142 & \\
\hline \multicolumn{2}{|c|}{ Number of migraine days during control month } & -0.538 & & 0.002 & \\
\hline
\end{tabular}

\begin{tabular}{lrrrc}
\hline & \multicolumn{1}{c}{$B$} & SE & \multicolumn{1}{l}{$\beta$} & \multicolumn{1}{c}{$P$} \\
\hline Migraine years & 0.08 & 0.02 & 0.56 & 0.002 \\
Throbbing headache & -0.73 & 0.32 & -0.31 & 0.032 \\
Aura & 0.88 & 0.38 & 0.33 & 0.03 \\
Married & -2.38 & 0.58 & -0.69 & $<0.001$ \\
Number of migraine days during the control month & -0.43 & 0.08 & -0.77 & $<0.001$ \\
\hline
\end{tabular}

The drug should be discontinued immediately after the end of the Ramadan (The Islamic holiday of Eid ul-Fitr). In addition, there is no available data regarding the use of beta-blockers and tricyclic antidepressants during prolonged fasting. The potential for weakness, lightheadedness and hypotension [10] caused by these drugs could be aggravated during fasting and dehydration, which makes them bad choices for fasting patients. Topiramate requires a long titration and a twice daily regimen.

Rofecoxib (Vioxx ${ }^{\circledR}$ ) was found [11] to be effective as a prophylactic treatment to Yom-Kippur headache. In addition, this drug is no longer available; other long-acting nonsteroidal antiinflammatory drugs can be used, taken immediately after an early breakfast prior to sunrise.

Similarly long-acting triptan, such as naratriptan can be used; also caution must be drawn to the development of chronic daily headache.

Another option is the use of long-acting valproic acid preparation, taken once daily. This drug was used [12] successfully in a patient with episodes of hypoglycemia followed by attacks of migraine without aura.

Future studies should address the limitations of this preliminary study, looking into the circadian preponderance of the attacks, their relation to menstruation, or intercurrent illness in larger groups of migraine sufferers and non-sufferers.

Further studies are also needed to find effective ways to identify patient at risk and ways to manage the Ramadanassociated migraine exacerbation, which affects the lives of millions of Muslim migraine patients world wide, and many of them exacerbate 1 month each year.

Conflict of interest None.

\section{References}

1. Sakr AH (1975) Fasting in Islam. J Am Diet Assoc 67(1):17-21

2. Bigal ME, Lipton RB (2009) The epidemiology, burden, and co morbidities of migraine. Neurol Clin 27(2):321-334

3. Miller T (ed) (2009) Mapping the global Muslim population: a report on the size and distribution of the world's Muslim population. Pew Research Center. http://pewforum.org/newassets/ images/reports/Muslimpopulation/Muslimpopulation.pdf. Accessed 02 October 2010

4. Torelli P, Evangelista A, Bini A, Castellini P, Lambru G, Manzoni GC (2009) Fasting headache: a review of the literature and new hypotheses. Headache 5:744-752

5. Headache Classification Committee of the International Headache Society (2004) The International Classification of Headache Disorders. Cephalalgia 24(Suppl 1):1-160 
6. Mosek A, Korczyn AD (1995) Yom Kippur headache. Neurology 45(11):1953-1955

7. Mosek A, Korczyn AD (1999) Fasting headache, weight loss, and dehydration. Headache 39:225-227

8. Awada A, Al Juman AI (1999) The First-of-Ramadan headache. Headache 39:490-493

9. Topacoglu H, Karcioglu O, Yuruktumen A et al (2005) Impact of Ramadan on demographics and frequencies of disease-related visits in the emergency department. Int J Clin Pract 59:900-905
10. Mosnaim AD, Abiola R, Wolf ME, Perlmuter LC (2010) Etiology and risk factors for developing orthostatic hypotension. Am J Ther 17(1):86-91

11. Drescher MJ, Elstein Y (2006) Prophylactic COX 2 inhibitor: an end to the Yom Kippur headache. Headache 46:1487-1491

12. Jacome DE (2001) Hypoglycemia rebound migraine. Headache $41: 895-898$ 